\title{
Identification and control using LSDP approach applied to the PT326 blower
}

\author{
MARWA YOUSFI \\ Research Laboratory of \\ Automatic Signal and Image \\ Processing \\ National Engineering School \\ of Monastir, University of \\ Monastir, \\ Ibn ELJazzar Street, 5019 \\ Monastir, TUNISIA
}

\author{
TAREK GARNA \\ Higher Institute of Applied \\ Science and Technology of \\ Sousse, \\ University of Sousse, \\ Ibn Khaldoun city, 4003 \\ Sousse, TUNISIA
}

\author{
CHAKIB BEN NJIMA \\ Higher Institute of Transport \\ and Logistics of Sousse, \\ University of Sousse \\ Erriadh city BP 247, 4023 \\ Sousse, TUNISIA
}

Abstract - The present work focuses on the control of the nonlinear system, the PT326 blower, using the LSDP (Loop Shaping Design Procedure) approach in the discrete case and the gain scheduling technique. First, the system's behavior is described by defining two operating points. In each of these latter the system is described using the linear ARX (Auto Regressive model with Xternal inputs) model which we propose to identify its parameters using the least squares method. Then, for each operating point the LSDP approach is exploited to synthesize a local robust controller. Later, the global robust controller resulting from switching between the local robust controllers by exploiting the gain scheduling technique is used to control the nonlinear PT326 blower.

Key Words: ARX model, LSDP approach, gain scheduling

Received: June 27, 2020. Revised: October 19, 2020. Accepted: December 4, 2020. Published: December 31, 2020.

\section{Introduction}

The PT326 process trainer presented in Figure 1, models industrial situations when the temperature control is required in the presence of transport delay. The process involves air transported and heated as it passes over a heater grid before being released into the atmosphere through a tube. The control objective is to maintain the temperature of the air $y$ at a desired level. Temperature control is achieved by varying the electrical power $u$ supplied to the heater grid . The air temperature may be sensed by using a bead thermistor placed in the flow at any of three positions along the tube. The spatial separation between the thermistor and the heater introduces a transport delay into the system [3].

Generally the model identification is determining the parameters of a mathematical model, the structure is established according to a given criterion, the model parameters are obtained by minimizing the prediction error between the measured output and the estimated output according an optimality criterion (e.g., least squares, mean square error, maximum likelihood), we are particularly interested in the least squares method. The goal is to find the best set of parameters $\theta$, which from a set of measurements $\varphi$ predicts with accuracy the output actually measured. Suppose that as a result of an experience we have

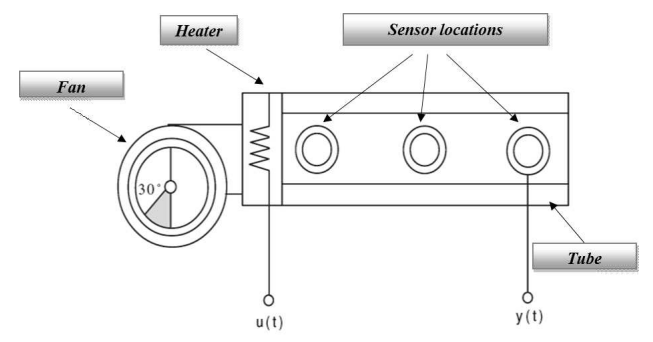

Fig. 1. the PT326 blower bloc diagram a set of measures $\{y(k), u(k) ; k=1, \ldots, M\}$ and the set of parameters $\theta$ is unknown. We construct the sequence of the predicted output:

$$
y(k)=\theta(k)^{T} \varphi(k)
$$

The vector of parameters $\theta$ is the one that minimizes the following norm:

$$
\theta=\arg \max (Q N)=\arg \max \left(\frac{1}{M} \sum_{k=1}^{M}\left(y(k)-\theta(k)^{T} \varphi(k)\right)^{2}\right)
$$

So we get the classical least square solution in its recursive form (RLS: Recursive Least Square):

$$
\theta(k)=\theta(k-1)+F(k) \varphi(k)\left(y(k)-\theta^{T}(k-1) \varphi(k)\right)
$$

where:

$$
F(k)^{-1}=F(k-1)^{-1}+\varphi(k) \varphi(k)^{T}
$$

and

$$
F(k)=F(k-1)-\frac{F(k-1) \varphi(k) \varphi(k)^{T} F(k-1)}{1+\varphi(k)^{T} F(k-1) \varphi(k)}
$$

Consequently in order to apply the RLS technique for the identification of the PT326 blower we propose to identify the 
system parameters in two operating points. This can be done by applying two different input sequences to the system and recovering the corresponding output signals for each operating point. Each of these latter is represented by a strictly causal discrete time linear system described by an ARX model [1]:

$$
y(k)=\sum_{j=1}^{n a} h_{a}(j) y(k-j)+\sum_{j=1}^{n b} h_{b}(j) u(k-j)
$$

where $u(k)$ and $y(k)$ are the system input and output respectively, $n a$ and $n b(n b<n a)$ are the model orders associated to the output and the input respectively, $h_{a}$ and $h_{b}$ are the model parameters. The ARX model (6) can be rewritten as a vector form as following:

$$
y(k)=\theta^{T} \varphi
$$

where $\varphi$ is the regression vector and $\theta$ is the parameters vector:

$$
\varphi=[y(k-1) \ldots y(k-n a) u(k-1) \ldots u(k-n b)]^{T}
$$

and

$$
\theta=\left[h_{a}(1) \ldots h_{a}(n a) h_{b}(1) \ldots h_{b}(n b)\right]^{T}
$$

In figures 2 and 3 , we present the input and output signals used for the identification of the PT326 blower for each considered operating point. The used input signals are random gaussian signals. We resume, in table I, the identification results for each operating point illustrated.
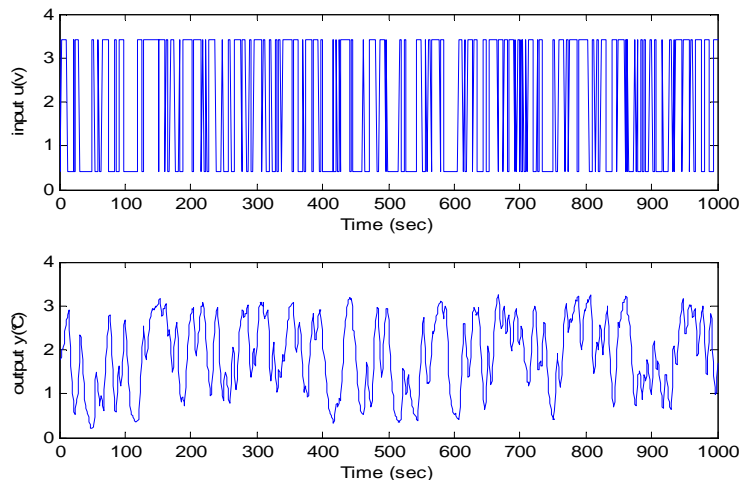

Fig. 2. First operating point input and output signals
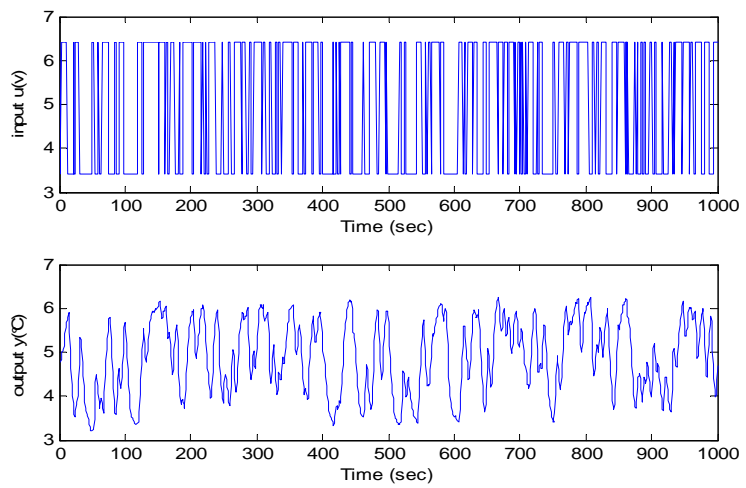

Fig. 3. Second operating point input and output signals
TABLE I

IDENTIFICATION RESULTS OF THE PT326 PROCESS TRAINER

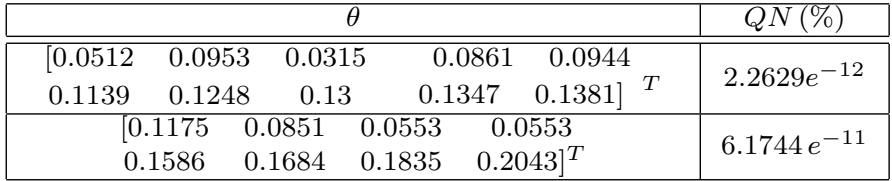

\section{Linear Robust Controller Using LSDP Approach}

\subsection{Control Principle by Discrete Loop Shaping}

The loop shaping technique [5] consists in using a precompensator or a weighting filter $V_{c}(s)$ to ameliorate the performance of open loop continuous transfer function $G_{c}(s)$ as illustrated in Figure 4. Typically $V_{c}(s)$ aims to ensure a high gain at low frequencies (to ensure a zero static error in the case of a tracking reference) and low gain at high frequencies (to ensure robustness against the disturbance rejection at high frequencies).

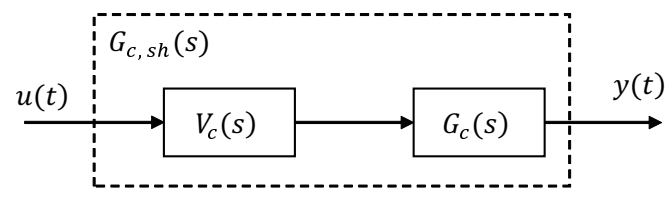

Fig. 4. Continuous time loop shaping principle

This technique provides the commonly known shaped system given by:

$$
G_{c, s h}(s)=V_{c}(s) G_{c}(s)
$$

Our aim is to exploit the loop shaping technique in the discrete case to identify the discrete weighting filter $V_{d}(z)$ associated with the discrete shaped system defined as follows:

$$
V_{d}(z)=\frac{\overline{V_{c} G_{c}}(z)}{G_{d}(z)}
$$

For digital control of a continuous system, the discrete loop shaping is presented in Figure 5.

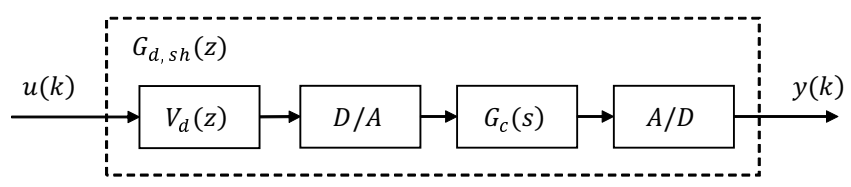

Fig. 5. Loop shaping in the discrete case

\subsection{Coprime Factor Uncertainty Representation}

The discrete shaped system $G_{d, s h}(z)$ defined by:

$$
G_{d, s h}(z)=V_{d}(z) G_{d}(z)
$$


can be written for an input $u(k)$ and an output $y(k)$ in the following state space representation [2]:

$$
\left\{\begin{array}{l}
x(k+1)=A_{s h} x(k)+B_{s h} u(k) \\
y(k)=C_{s h} x(k)+D_{s h} u(k)
\end{array}\right.
$$

with $A_{s h} \in \Re^{n \times n}, B_{s h} \in \Re^{n \times 1}, C_{s h} \in \Re^{1 \times n}$ and $D_{s h} \in \Re$ such as pairs of state matrices $\left(A_{s h}, B_{s h}\right)$ and $\left(A_{s h}, D_{s h}\right)$ are respectively controllable and observable, $n \in N^{+}$is the order of the system. In this case, the shaped system can be written in the following forms:

$$
\begin{gathered}
G_{d, s h}(z):=\left(\begin{array}{l|l}
A_{s h} & B_{s h} \\
\hline C_{s h} & D_{s h}
\end{array}\right) \\
G_{d, s h}(z)=D_{s h}+C_{s h}\left(z I_{n}-A_{s h}\right)^{-1} B_{s h}
\end{gathered}
$$

where $G_{d, s h}(z) \in \Re H_{\infty}$ the space of proper and real rational stable transfer functions. The LSDP approach is based on the configuration depicted in Figure 6, where:

$$
G_{d, s h}(z)=\tilde{M}_{d, s h}^{-1}(z) \tilde{N}_{d, s h}(z)
$$

with $\tilde{M}_{d, s h}(z)$ and $\tilde{N}_{d, s h}(z)$ are the left normalized coprime factors of $G_{d, s h}(z)$ such that:

$$
\begin{aligned}
& \tilde{M}_{d, s h}(z):=\left(\begin{array}{c|c}
A_{s h}+R C_{s h} & R \\
\hline S^{-1 / 2} C_{s h} & S^{-1 / 2}
\end{array}\right) \\
& \tilde{N}_{d, s h}(z):=\left(\begin{array}{c|c}
A_{s h}+R C_{s h} & B_{s h}+R D_{s h} \\
\hline S^{-1 / 2} C_{s h} & S^{-1 / 2} D_{s h}
\end{array}\right)
\end{aligned}
$$

where:

$$
\begin{gathered}
R=-\left(B_{s h} D_{s h}+Y C_{s h}^{T}\right) S^{-1} \\
S=1+D_{s h}^{2}
\end{gathered}
$$

and $Y$ is the unique positive definite solution of the algebraic Riccati equation:

$$
\begin{gathered}
\left(A_{s h}-B_{s h} S^{-1} D_{s h}^{T} C_{s h}\right)^{T} Y+Y\left(A_{s h}-B_{s h} S^{-1} D_{s h}^{T} C_{s h}\right) \\
-Y C_{s h}^{T} S^{-1} C_{s h} Y+B_{s h} S^{-1} B_{s h}^{T}=0
\end{gathered}
$$

Subsequently, the LSDP approach makes it possible to take into account the parametric uncertainties of the shaped system $G_{d, s h}(z)$ by considering the representation in the form of left normalized coprime factorization (LNCF) to obtain the uncertain shaped system $G_{d, s h}^{\Delta}(z)$ :

$G_{d, s h}^{\Delta}(z)=\left(\tilde{M}_{d, s h}(z)+\Delta_{s h, \tilde{M}}(z)\right)^{-1}\left(\tilde{N}_{d, s h}(z)+\Delta_{s h, \tilde{N}}(z)\right)$

where $\Delta_{s h, \tilde{M}}(z)$ and $\Delta_{s h, \tilde{N}}(z)$ are the parametric uncertainties over the left normalized coprime factors, they are unknown stable transfer functions, but bounded in infinity norm.

To maximize the class of perturbed models such that the closed-loop system in Figure 6, is stabilized by a controller $K_{\infty}(z)$, this latter must stabilize the nominal plant $G_{d}$ and maximize $\varepsilon$ where:

$$
\varepsilon^{-1}=\left\|\left[\begin{array}{c}
K_{\infty} \\
1
\end{array}\right]\left(1-G_{d, s h} K_{\infty}\right)^{-1} \tilde{M}_{d, s h}^{-1}(z)\right\|_{\infty}
$$

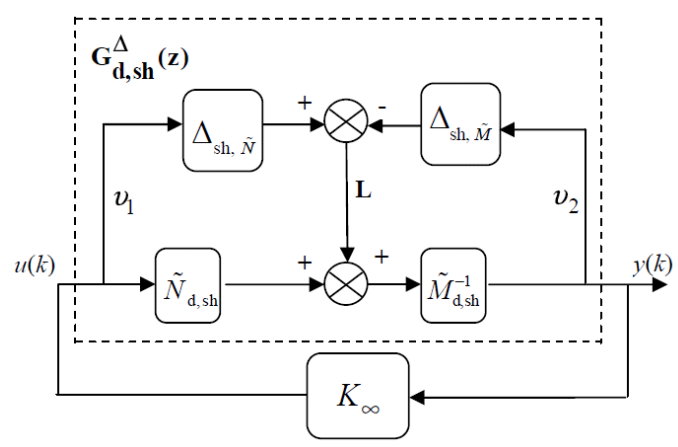

Fig. 6. Robust stabilization principle

From the small gain theorem, the closed-loop system will remain stable if:

$$
\left\|\left[\Delta_{s h, \tilde{N}} \Delta_{s h, \tilde{M}}\right]\right\|_{\infty}<\varepsilon
$$

The maximum stability margin is derived by McFarlane and Glover in [5] as:

$$
\varepsilon_{\max }=\left(1+\lambda_{\max }(X Y)\right)^{-1 / 2}
$$

where $\lambda_{\max }(X Y)$ is the maximum eigenvalue of the product matrix $X Y$ and $Y$ is the positive-definite solution of the algebric Riccati equation given in (20) and $X$ is the solution of the following algebric Riccati equation:

$$
\begin{array}{r}
\left(A_{s h}-B_{s h} S^{-1} D_{s h} C_{s h}\right)^{T} X+X\left(A_{s h}-B_{s h} S^{-1} D_{s h} C_{s h}\right) \\
-X B_{s h} S^{-1} B_{s h}^{T}+C_{s h}^{T} S^{-1} C_{s h}=0
\end{array}
$$

A controller which achieves both robust stability and $\varepsilon_{\max }$ is given by:

$$
\begin{aligned}
& K_{\infty, \text { opt }}(z):= \\
& \left(\frac{A_{s h}+B_{s h} F+\varepsilon_{\max }^{-2}\left(L^{T}\right)^{-1} Y C_{s h}^{T}\left(C_{s h}+D_{s h} F\right)}{B_{s h}^{T} X} \mid\right. \\
& \left.\frac{\varepsilon_{\max }^{-2}\left(L^{T}\right)^{-1} Y C_{s h}^{T}}{-D_{s h}^{T}}\right)
\end{aligned}
$$

where:

$$
\begin{gathered}
F=-S^{-1}\left(D_{s h} C_{s h}+B_{s h}^{T} X\right) \\
L=\left(1-\varepsilon_{\max }^{-2}\right) I_{n \times n}+X Y
\end{gathered}
$$

To ensure the similarity between the frequency response of $\left(K_{\infty, o p t} G_{s h}\right)$ and that of $G_{s h}$ the value of $\varepsilon_{\max }$ must satisfy the following condition:

$$
0.2<\varepsilon_{\max }<1
$$

If the condition (29) is not satisfied, the weighting filter $V_{c}(s)$ have to be readjusted.

Finally, the final robust controller is calculated as follows:

$$
K_{d, f}(z)=V_{d}(z) K_{\infty, o p t}(z)
$$




\section{Synthesis of a Nonlinear Robust Controller}

In this section, we will be analyzing the behavior of the nonlinear system PT326 process trainer and proposing a method to synthesize a control law to ensure reference tracking performance. To do this we propose to decompose the representation of the nonlinear system PT326 into a set of linear sub-models according to the variation of a sequencing parameter. Then, the discrete LSDP approach is applied for each sub-model. Subsequently, the obtained final robust controllers serves to synthesize the global robust controller using the gain scheduling technique [4]. The obtained global robust controller is used to control he nonlinear system.

\subsection{Control by Gain Scheduling Technique}

Many industrial systems are characterized by variations on parameters that affect their dynamics. Calculate a controller for this type of systems is not an easy task because these variations must be taken into account to obtain efficient control laws. One of the techniques used to overcome these constraints is the control by gain scheduling technique to build an adaptive control that count for parameter variations. This method has many advantages such as the ability to use the tools of modern theory of robust control and also the ability to update the parameters of the correction block.

The control by gain scheduling is based on the calculation of its coefficients off-line for several operating conditions of the system, and then adapts these coefficients using interpolation methods. The interpolation/ adaptation is made from a set of parameters varying in time that captures the change of system's operating conditions, which is often called scheduling parameter. For this technique, a set of linear invariant representation (LTI) is calculated, the a controller is calculated for each LTI representation using such powerful modern techniques of robust control theory, ex LSDP approach, that meets the performance requirements of the user locally. Finally, a nonlinear global controller is calculated by combining these local controllers through an interpolation method with respect to the scheduling parameter measured in real time.

\subsection{Synthesis of a Robust Controller for the PT326 Process Trainer}

1) Synthesis of the weighting controller: In our study we will use two operating points to describe the nonlinear behavior of the system, depending on the temperature variation which represents the scheduling parameter.

$$
S:\left\{\begin{array}{l}
G_{d}^{1}(z) \text { if } \quad 0.5^{\circ} \mathrm{C} \leq y<3.3^{\circ} \mathrm{C} \\
G_{d}^{2}(z) \text { if } 3.3^{\circ} \mathrm{C} \leq y \leq 6.3{ }^{\circ} \mathrm{C}
\end{array}\right.
$$

Each linear model $G_{d}^{j}(z), j=1,2$ is defined for a specific operating point where the identified parameters are presented in table I. To establish the shaped representation $G_{c, s h}(s)$, the chosen weighting filter is given as follows:

$$
V_{c}(s)=\frac{5}{s+0.01}
$$

In the discrete case we integrate a zero-order holder circuit with a sampling period $T_{e}=50 \mathrm{~ms}$.

In equation (11), $G_{d}(s)$ is replaced by $G_{d}^{j}(z), j=1,2$ to deduce the discrete weighting filter associated to $G_{d}^{j}(z), j=$ 1,2 :

$$
\begin{gathered}
V_{d}^{1}(z)=\frac{0.01499 z^{6}+0.01267 z^{5}-0.003508 z^{4}-0.003743 z^{3}}{0.0512 z^{6}-0.001058 z^{5}-0.1027 z^{4}+0.1109 z^{3}} \\
\frac{-0.003888 z^{2}-0.004007 z-0.001985}{-0.03986 z^{2}-0.1017 z+0.08327} \\
V_{d}^{2}(z)=\frac{0.01499 z^{5}+0.012 z^{4}-0.004805 z^{3}-0.005172 z^{2}}{0.1175 z^{5}-0.136 z^{4}-0.001216 z^{3}-0.001811 z^{2}} \\
\frac{-0.005701 z-0.002937}{-0.002413 z+0.02399}
\end{gathered}
$$

with $G_{d}^{j}(z), j=1,2$ are given by:

$$
\begin{aligned}
& G_{d}^{1}(z)=\frac{0.0512 z^{4}+0.0953 z^{3}+0.0315 z^{2}+0.0861 z+0.0944}{z^{5}-0.1139 z^{4}-0.1248 z^{3}-0.13 z^{2}-0.1347 z-0.1381} \\
& G_{d}^{2}(z)=\frac{0.1175 z^{3}+0.0851 z^{2}+0.0553 z+0.0272}{z^{4}-0.1586 z^{3}+0.1684 z^{2}+0.1835 z+0.2043}
\end{aligned}
$$

Therefore we calculate the shaped system corresponding for each operation point:

$$
\begin{aligned}
G_{d, s h}^{1}(z)=\frac{0.006525 s^{6}+0.01265 s^{5}-0.000575 s^{4}+0.001 s^{3}+}{s^{7}-1.996 s^{6}+0.9716 s^{5}+0.0044 s^{4}-0.0001 s^{3}+} \\
\frac{0.009475 s^{2}-0.008375 s-0.01019}{0.0007 s^{2}+0.1411 s-0.1218} \\
G_{d, s h}^{2}(z)=\frac{0.015 s^{5}+0.012 s^{4}-0.0048 s^{3}-0.005175 s^{2}-}{s^{6}-2.041 s^{5}+1.012 s^{4}-0.0065 s^{3}-0.0075 s^{2}+} \\
\frac{0.0057 s-0.002938}{0.2226 s-0.1802}
\end{aligned}
$$

The result of the simulation under Simulink of the shaped system is given in Figures 7 and 8, where we represent the output and reference signals and control signal respectively.

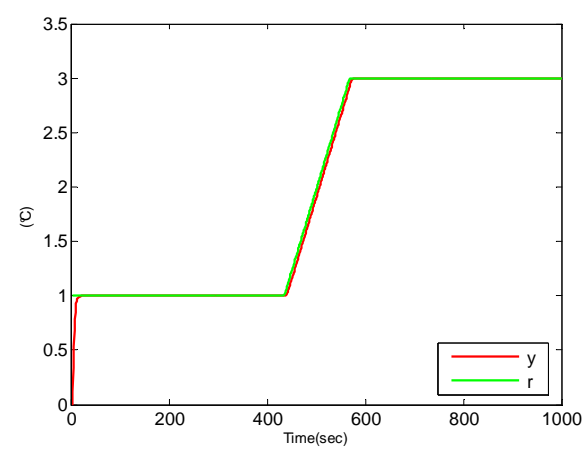

Fig. 7. Output $y$ and reference $r$ signals of the shaped system 


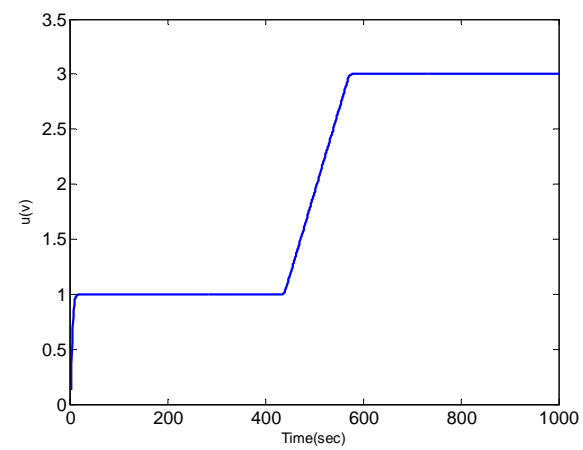

Fig. 8. Control signal $u$ of the shaped system

2) Synthesis of robust controllers: After all calculation we get the maximum stability margin $\varepsilon_{\max }$ for each operating point:

$$
\varepsilon_{\max }^{1}=0.4795, \quad \varepsilon_{\max }^{2}=0.5043
$$

By applying the LSDP approach, arobust controller $K_{\infty, o p t}$ is calculated for each operating point:

$$
\begin{array}{cc}
K_{\infty, \text { opt }}^{1}= & e^{-2} \frac{-21.54 z^{7}+21.26 z^{6}+0.5492 z^{5}+0.4548 z^{4}}{z^{7}-1.641 z^{6}+0.6605 z^{5}-0.007911 z^{4}} \\
K_{\infty, \text { opt }}^{2}= & \frac{+0.4582 z^{3}+0.4431 z^{2}-2.596 z+1.082 e^{-13}}{-0.01245 z^{3}-0.01356 z^{2}+0.1278 z-0.08419} \\
& \begin{array}{c}
-22.34 z^{5}+23.06 z^{4}+0.6663 z^{3}+0.8123 z^{2} \\
z^{6}-1.678 z^{5}+0.6781 z^{4}-0.02375 z^{3} \\
-0.02754 z^{2}+0.1992 z-0.1237
\end{array}
\end{array}
$$

Then, the final robust controllers are calculated:

$$
\begin{gathered}
K_{d, f}^{1}=\frac{-3.23 z^{9}+2.94 z^{8}+3.06 z^{7}-2.623 z^{6}+4.22 z^{5}}{1.175 z^{9}-4.139 z^{8}+5.441 z^{7}-0.3171 z^{6}+0.06875 z^{5}} \\
+1.399 z^{4}-4.423 z^{3}-8.601 z^{2}+3.294 z-1.373 \\
+3.413 z^{4}+0.01672 z^{3}-0.03955 z^{2}+0.03186 z-0.008726 \\
K_{d, f}^{2}=\frac{-3.349 z^{7}+3.201 z^{6}+3.199 z^{5}-2.796 z^{4}}{0.1175 z^{8}-0.4183 z^{7}+0.5543 z^{6}-0.3266 z^{5}} \\
\quad+7.203 z^{3}-0.06896 z^{2}-0.01704 z+0.05059 \\
+0.07229 z^{4}+0.02704 z^{3}-0.06144 z^{2}+0.048 z-0.01282
\end{gathered}
$$

We present in Figures 9 to 12, the results of the validation of the calculated robust controller for each operating point.

The control of nonlinear system by the gain scheduling technique can be illustrated by equation (32) and Figure 13 , where the global robust controller $K_{\infty}^{\text {global }}$ of the PT326 is constructed by switching between the local controllers $K_{\infty, \text { opt }}^{j}, j=1,2$ depending on the selection signal $I_{s}$ of the function Fcn controlling the switching of the switcher according to the value of the sequencing parameter $y$.

$$
\left\{\begin{array}{l}
I_{1}=1 \text { and } K_{\infty}^{\text {global }}=K_{d, f}^{1} \text { if } \quad 0.5 \leq y \leq 3.3 \\
I_{2}=2 \text { and } K_{\infty}^{\text {global }}=K_{d, f}^{2} \text { if } \quad 3.3<y \leq 6.3
\end{array}\right.
$$

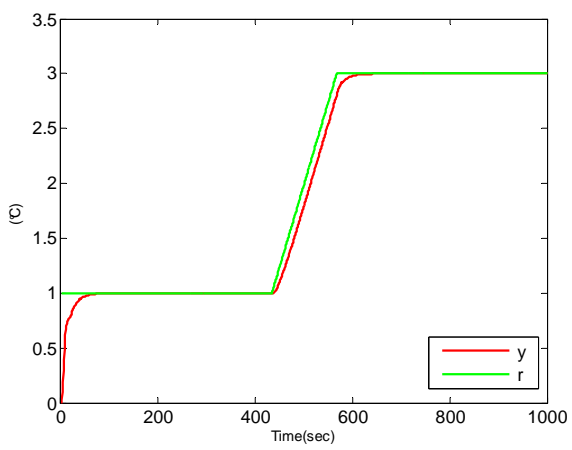

Fig. 9. Validation of the robust controller $K_{\infty, o p t}^{1}$

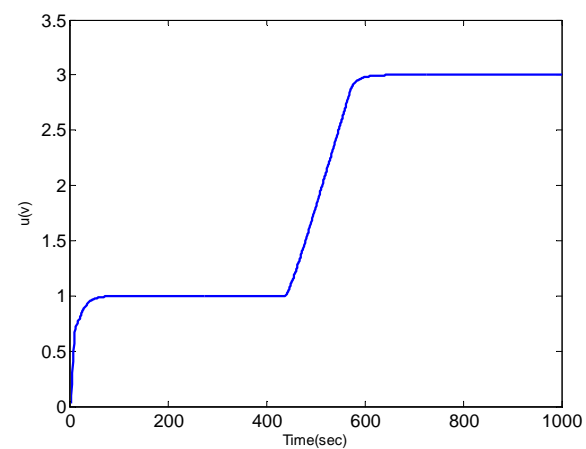

Fig. 10. Control signal $u$ for the first operating point

To validate the global robust controller, we present in Figures 14, 15 and 16 the evolution of reference and output signals, control signal and selection signal respectively.

Figure 14 shows that the output signal $y(k)$ follows the evolution of the reference signal $r(k)$ for both operating points. This highlights the good performance of the global robust controller function of the two robust controllers $K_{\infty, \text { opt }}^{j}, j=1,2$. These latter are involved depending on the temperature variation which represents the scheduling parameter as described

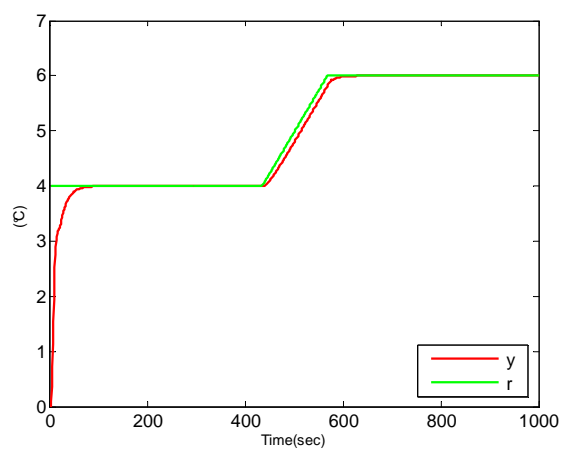

Fig. 11. Validation of the robust controller $K_{\infty, \text { opt }}^{2}$ 


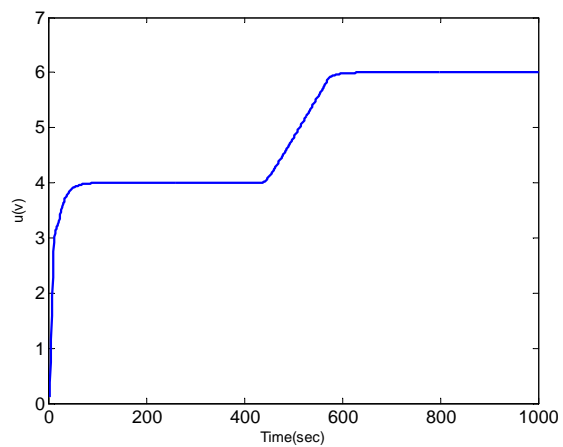

Fig. 12. Control signal $u$ for the second operating point

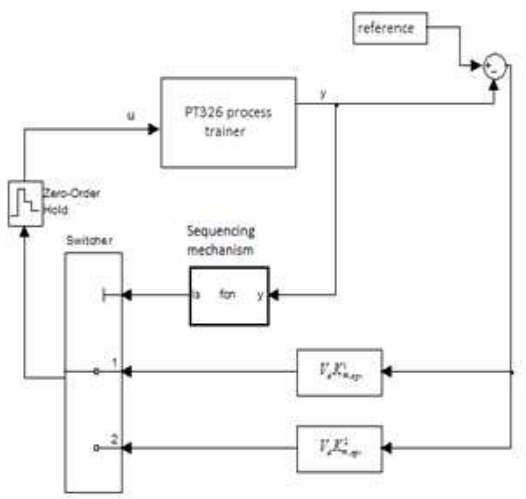

Fig. 13. Global control by gain scheduling

in (32). This variation is illustrated by Figure 16, where the selection signal $I_{s}$ switch between the two robust controllers.

To test the robustness of the global robust controller facing parametric uncertainties, we propose to disrupt the system by changing the value of the resistance of the heater. The result, presented in Figure 17, shows that the output track well the reference even in presence of parametric uncertainty. We present in Figure 18 the result of the simulation of a PID controller for the same conditions. The result proves the

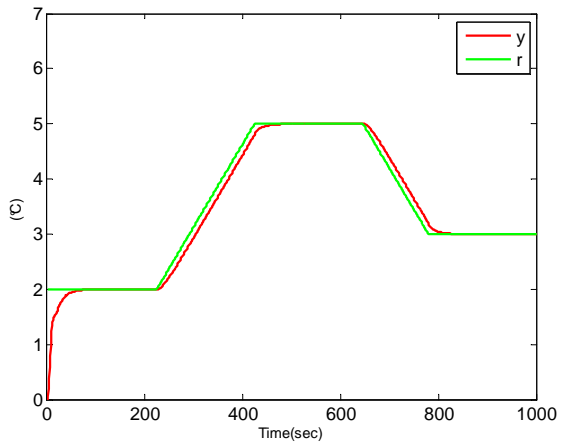

Fig. 14. Reference and output signals associated with $K_{\infty}^{\text {global }}$

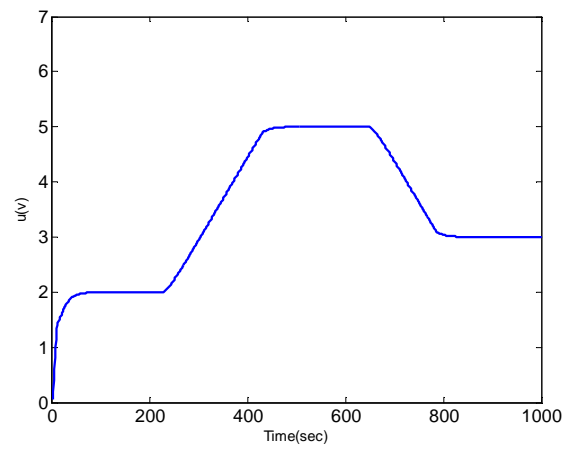

Fig. 15. Control signal associated with $K_{\infty}^{\text {global }}$

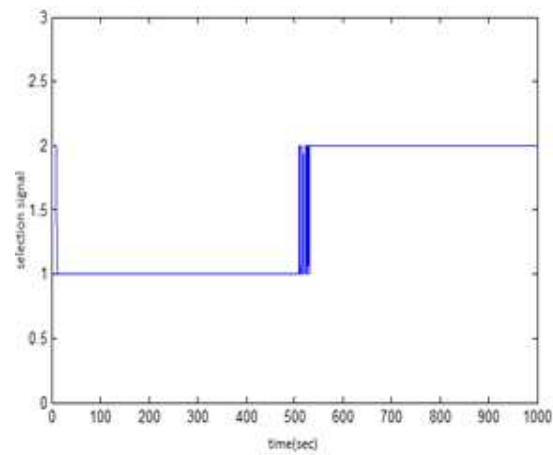

Fig. 16. Selection signal associated with $K_{\infty}^{\text {global }}$

superiority of the robustness of the global robust controller compared to the PID controller.

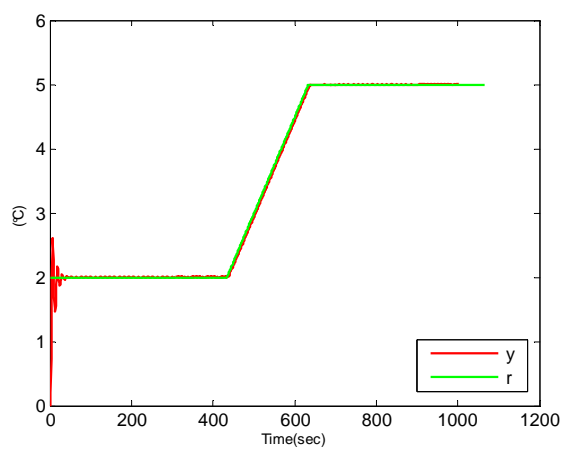

Fig. 17. Robustness test of the global robust controller

\section{Conclusion}

In this paper we used the LSDP of McFarlane and Glover (1990) combined with the gain scheduling technique to synthetize a robust controller for a nonlinear system, by dividing its operating domain to two point. This was done in 5 steps 1) Using a weighting filter to ameliorate the system's open loop performances. 2) Representation of the shaped system's uncertainties as LNCF. 3) Synthesis of a feedback controller which 


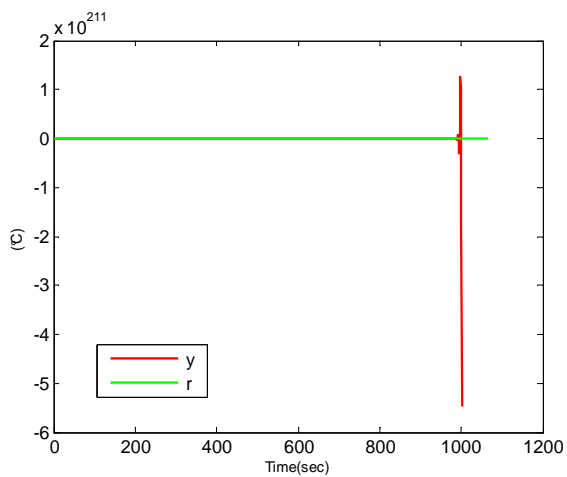

Fig. 18. Robustness test of the PID controller

robustly stabilizes the LNCF of the shaped system for each operating point. 4) The final robust controllers are calculated by combining the weighting filters and the robust controllers. 5) Finally the global robust controller is constructed using the gain scheduling technique by switching between the final robust controllers.

\section{References}

[1] [1] Bouzrara K, Garna T, Ragot J, et al. (2013) Online identification of the ARX model expansion on Laguerre orthonormal bases with filters on model input and output. International Journal of Control 86(3): 369-385.

[2] [2] Haj Salah A Ameur, Garna T, Ragot J, Hassani M. Synthesis of a robust controller with reduced dimension by the Loop Shaping Design Procedure and decomposition based on Laguerre functions Transactions of the Institute of Measurement and Control 1-25

[3] [3] Mohd. fua'ad Rahmat, Yeoh Keat Hoe, Sahnius Usman and Norhaliza Abdul Wahab. Modelling of pt326 hot air blower trainer kit using prbs signal and cross-correlation technique Transactions of Jurnal Teknologi, 42(D) Jun. 2005: 9-22

[4] [4] Rugh W.J. et Shamma J.S., Research on Gain Scheduling. Automatica, Vol. 36, pp. 1401-1425, 20

[5] [5] McFarlane D. et Glover K., Robust Controller Design Using Normalized Coprime Factor Plant Descriptions. Vol. 138 of Lecture Notes in Control and Information Sciences, Springer-Verlag, Berlin, 1990.

\section{Creative Commons Attribution License 4.0 (Attribution 4.0 International, CC BY 4.0)}

This article is published under the terms of the Creative Commons Attribution License 4.0

https://creativecommons.org/licenses/by/4.0/deed.en US 